\title{
CHROMATIX: computing the functional landscape of many-body chromatin interactions in transcriptionally active loci from deconvolved single cells
}

\author{
Alan Perez-Rathke ${ }^{1}$, Qiu Sun ${ }^{2}$, Boshen Wang ${ }^{1}$, Valentina Boeva ${ }^{3,4}$, Zhifeng Shao ${ }^{5}$ and Jie Liang ${ }^{1 *}$ (D)
}

\begin{abstract}
Chromatin interactions are important for gene regulation and cellular specialization. Emerging evidence suggests many-body spatial interactions play important roles in condensing super-enhancer regions into a cohesive transcriptional apparatus. Chromosome conformation studies using $\mathrm{Hi}-\mathrm{C}$ are limited to pairwise, population-averaged interactions; therefore unsuitable for direct assessment of many-body interactions. We describe a computational model, CHROMATIX, which reconstructs ensembles of single-cell chromatin structures by deconvolving Hi-C data and identifies significant many-body interactions. For a diverse set of highly active transcriptional loci with at least 2 super-enhancers, we detail the many-body functional landscape and show DNase accessibility, POLR2A binding, and decreased H3K27me3 are predictive of interaction-enriched regions.
\end{abstract}

Keywords: Chromatin folding, Polymer modeling, Super-enhancers, Topologically associated domains, Epigenomics, Histone modifications, Sequential Monte Carlo sampling, Gibbs sampling, Bayesian inference, Machine learning, CHROMATIX

\section{Background}

Chromosome folding and nuclear organization play essential roles in fundamental processes such as regulation of gene expression $[1,2]$ and cellular specialization $[3,4]$. A wealth of information on chromatin organization has been gained through studies based on chromosome conformation capture techniques such as Hi-C [5-8], which measure pairwise, proximity interactions between chromatin regions that are averaged over a population of cells $[6,9]$. There is now growing evidence that multivalent interactions play important roles in formation of phase-separated and highly dense, functional chromatin assemblies in super-enhancers (SEs) [10, 11]; however, it is difficult to detect and quantify many-body $(\geq 3)$ interactions from pairwise and averaged $\mathrm{Hi}-\mathrm{C}$ measurements.

${ }^{*}$ Correspondence: jliang@uic.edu

${ }^{1}$ Richard and Loan Hill Department of Bioengineering, University of Illinois at Chicago, Chicago, IL, USA

Full list of author information is available at the end of the article
Several experimental techniques have been developed to detect putative many-body chromatin interactions. These include single-cell Hi-C [12-14], Dip-C [15, 16], Tri-C [2], GAM [17], and SPRITE [18]. However, there are limitations with these techniques. For example, while single-cell Hi-C permits detection of instances of manybody interactions in individual cells, it often has low genomic coverage [19]; GAM and SPRITE do not readily distinguish direct from indirect many-body chromatin interactions due to ancillary coupling effects $[17,18]$. Overall, our current knowledge of many-body chromatin interactions and their functional roles in chromatin condensation is limited.

With the extensive availability of population-averaged Hi-C data for many biological systems, we ask whether it is possible to gain insight into functionally important many-body spatial interactions from these highquality, high-resolution measurements. While no computational method is currently available, we hypothesize that 3-D polymer modeling can be used to overcome the limitations of population-averaged, pairwise 
$\mathrm{Hi}-\mathrm{C}$ measurements. However, there are a number of significant technical challenges. These include (i) deconvolving the population-averaged and pairwise $\mathrm{Hi}-\mathrm{C}$ contact frequencies into an underlying ensemble of singlecell 3-D chromatin folds, such that instances of manybody interactions in single cells are collectively consistent with the input $\mathrm{Hi}-\mathrm{C}$, and (ii) distinguishing specific (i.e., highly non-random) many-body interactions from nonspecific interactions which are largely due to effects of linear genomic proximity [20] and nuclear confinement [21-23].

Modeling of 3-D chromatin structure allows for detailed analysis of nuclear organization patterns and can detect spatially interacting regions [21-34]. There are many well-developed physical models for chromatin folding, including the Strings and Binders Switch (SBS) model [24], the Minimal Chromatin Model (MiChroM) [26, 28], and the n-Constrained Self-Avoiding Chromatin (nCSAC) model $[21,22]$. The nCSAC approach folds polymers under the influence of predicted specific pairwise interactions obtained after controlling for effects of nuclear confinement. The SBS and MiChroM models follow block copolymer approaches [29, 30], in which chromatin regions are assigned different affinities for each other based on their corresponding types. In SBS, chromatin types are defined by their affinity to Brownian binder particles which facilitate bridging of multiple chromatin sites up to a specified valency. In MiChroM, chromatin types and affinities are based on clustering of epigenetic markers, followed by maximumentropy optimization of the resulting energy function. SBS and MiChroM can reproduce important physical phenomena such as the dynamics of chromatin condensation leading to phase separation; however, no methods for calling specific many-body chromatin interactions based on these models have been reported yet.

Several computational methods have been developed to detect specific pairwise chromatin interactions present within $\mathrm{Hi}-\mathrm{C}$ datasets [20]. These include the negative binomial model of Jin et al. [35], the non-parametric spline approach of Fit-Hi-C [36], the binomial model of GOTHiC [37], the local neighborhood loop-calling approach of HiCCUPS [9], and the hidden Markov random field model of $\mathrm{Xu}$ et al. [38]. These methods rely on the empirical $\mathrm{Hi}-\mathrm{C}$ for estimation of a background model that is then used to assess the significance of each pairwise chromatin contact; hence, these approaches may contain intrinsic bias as the observed $\mathrm{Hi}-\mathrm{C}$ data is being used for construction of its own null hypothesis test. In addition, these methods lack a 3-D folding model and therefore cannot assess the significance of many-body $(\geq 3)$ chromatin spatial interactions.
In this work, we describe CHROMATIX (CHROMatin mIXture), a new computational approach for detecting specific many-body interactions from populationaveraged $\mathrm{Hi}-\mathrm{C}$ data. We focus on uncovering occurrences where 3,4 , or more genomic regions all spatially co-locate to within a defined Euclidean distance threshold. We further require that these occurrences do not arise from simple physical effects of monomer connectivity, excluded volume, and spatial confinement; we refer to these as specific many-body interactions.

We extend the nCSAC $[21,22]$ folding method which allows for nearly unbiased construction of random polymer chains to serve as a null model completely decoupled from the Hi-C data. By further integrating extensive polymer simulations under a Bayesian generative framework [39], we resolve complex dependencies among chromatin contacts and deconvolve population $\mathrm{Hi}-\mathrm{C}$ data into the most likely single-cell contact states. These contact states are then folded to produce a 3-D structural ensemble consistent with the measured $\mathrm{Hi}-\mathrm{C}$. We achieve our results through a novel deep-sampling algorithm called fractal Monte Carlo, which can generate 3-D polymer ensembles with improved structural diversity and target distribution enrichment (see Additional file 1: Supplementary Information).

To study highly non-random and direct higher-order interactions among super-enhancers, enhancers, and promoter regions, we apply our method to a diverse set of 39 highly transcriptionally active loci in the GM12878 mammalian cell line; specifically, all TADbounded [40, 41] loci $(<2 \mathrm{MB})$, each with at least 2 super-enhancers [1, 3, 4] showing evidence of possible super-enhancer condensation (see Additional file 1: Supplementary Information, Additional file 2: Table S1) [18]. We detect specific many-body interactions in each of these loci, summarize the landscape of functional associations among participating regions, and report common biological factors predictive of interaction enrichment.

\section{Results}

\section{Model for chromatin folding}

We independently modeled the 39 genomic loci, ranging in size from $480 \mathrm{~KB}$ to $1.94 \mathrm{MB}$, each as a connected, self-avoiding polymer chain where monomer beads represent $5 \mathrm{~KB}$ of $11-\mathrm{nm}$ chromatin fiber [42, 43]. Locus lengths in base pairs are from the corresponding TAD (arrowhead) boundaries as reported in Rao et al. [9] (see Additional file 1: Supplementary Information). Each locus was simulated under a confining sphere based on the GM12878 nuclear diameter reported in Sanborn et al. [44] and scaled to preserve a constant base pair density $\left(\mathrm{bp} / \mathrm{nm}^{3}\right)$. 


\section{Identifying specific interactions from $\mathrm{Hi}-\mathrm{C}$ data}

The CHROMATIX modeling pipeline is illustrated in Fig. 1. Briefly, we first identify pairwise specific contacts from measured $\mathrm{Hi}-\mathrm{C}$ interaction frequencies by following the general approach of Gürsoy et al. [21]; namely, we identify chromatin interactions with $\mathrm{Hi}-\mathrm{C}$ frequencies unlikely to be observed under a uniform random folding environment [45, 46]. We extend the approach of Gürsoy et al. by using the method of fractal Monte Carlo weight enrichment (see Additional file 1: Supplementary Information) to uniform randomly sample an ensemble of $\sim$ 400, 000 3-D polymer conformations (see Fig. 1a, and Additional file 1: Figure S1 for examples of random polymers). These polymers are used as a null ensemble for identifying significant $\mathrm{Hi}-\mathrm{C}$ interactions that are unlikely to be formed due to random chance (Fig. 1b). The assumption of spherical confinement makes this null model more stringent in calling specific interactions as discussed in [22], although our tool supports other confinement models (e.g., ellipsoid). Details on $p$ value calculations can be found in the "Methods" section.

\section{Identifying a minimal set of sufficient interactions}

We conjecture that not all specific interactions are required to produce the observed $\mathrm{Hi}-\mathrm{C}$ chromatin folding patterns [22, 47]. To identify a minimal set of interactions that are sufficient to drive chromatin polymers into a folded ensemble that exhibit the observed $\mathrm{Hi}-\mathrm{C}$ frequencies, we retain roughly $5 \%$ of the identified specific contact interactions using clustering $[48,49]$ (see Additional file 1: Supplementary Information for more details). We call this procedure coarse-graining of the specific contacts (Fig. 1c); coarse-graining also regularizes our model to help prevent overfitting.

\section{Single-cell contact state deconvolution}

Many-body interactions occur probabilistically in individual cells. To reconstruct the 3-D chromatin polymer for each cell of a modeled population, we must predict which contacts among the set of minimally sufficient interactions are co-occurring within each individual cell. We call these co-occurring interactions the single-cell contact states (Fig. 1c). Once a single-cell contact state is properly generated, we then construct a set of 3-D chromatin polymers that are all consistent with this single-cell contact state. By generating a large number of single-cell contact states, we can obtain an ensemble of 3-D chromatin polymers which accurately reproduce the observed population $\mathrm{Hi}-\mathrm{C}$ measurements. Structural analysis of the ensemble of single-cell chromatin conformations can then reveal specific spatial many-body interactions.

The key to properly generating single-cell contact states is to account for dependencies among chromatin interactions; namely, how certain physical interactions may cooperatively induce formation of other interactions due to polymer folding. These dependencies are identified by in silico knock-in perturbation studies, where differential contact probabilities are assessed between two ensembles of chromatin polymers, one with and another without the target contact knocked-in. A large number of possible dependencies are identified through these extensive polymer knock-in simulations (see the "Methods" section and Additional file 1: Supplementary Information). Such simulations also identify geometrically infeasible contact combinations.

To properly deconvolve population $\mathrm{Hi}-\mathrm{C}$ interactions into single-cell contact states, we adopt a Bayesian generative approach. The dependencies and infeasible geometries among contacts are incorporated as a Bayesian

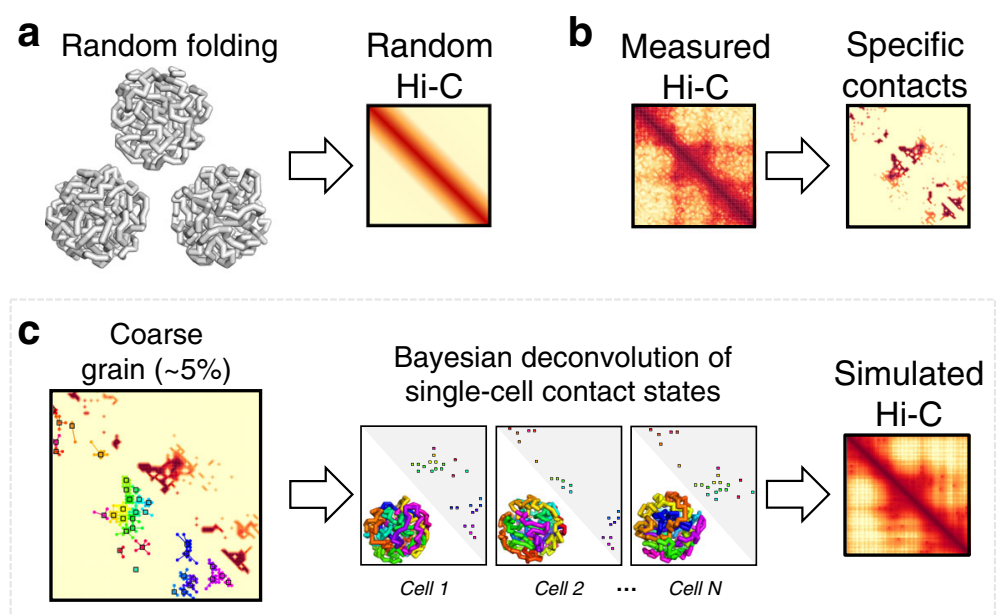

Fig. 1 CHROMATIX modeling pipeline. a Random polymers are generated using fractal Monte Carlo sampling. b Specific contacts are identified from measured Hi-C using a random polymer ensemble as the null distribution [21]. c Specific contacts are coarse-grained and single-cell contact states are deconvolved then folded to generate simulated Hi-C (see Additional file 1: Supplementary Information) 
prior. This physically based prior along with the measured $\mathrm{Hi}-\mathrm{C}$ data enables efficient Bayesian inference over the posterior distribution of single-cell contact states. Specifically, we use Gibbs sampling for this inference (see Additional file 1: Supplementary Information). For efficiency, we first coarse-grain the called specific $\mathrm{Hi}-\mathrm{C}$ interactions before carrying out knock-in simulations and Gibbs sampling. Only about $5 \%$ of the specific interactions are retained, which substantially reduces the computational cost, making this approach highly practical.

\section{Reconstructing 3-D chromatin folds}

For a given deconvolved single-cell state of chromatin contacts, we uniformly sample among the set of 3-D folds satisfying the spatial proximity interactions specified by the single-cell state. Specifically, we sample from the uniform distribution of chromatin chains conditioned on the deconvolved contact state of each cell, where two regions are spatially interacting if their Euclidean distance is $\leq 80 \mathrm{~nm}$ [47]. This procedure is repeated for each sampled single-cell contact state (see Additional file 1: Figure $\mathrm{S} 2$ for examples of sampled chromatin polymers).

Overall, we aggregate $\sim 50$ folds per single-cell to generate an ensemble of 25,000 3-D chromatin polymers at each of the 39 modeled genomic loci. These sampled conformations form the reconstructed ensemble of intrinsic 3-D folds underlying the population-aggregated $\mathrm{Hi}-\mathrm{C}$.

\section{Simulated 3-D polymer ensembles strongly correlate with $\mathrm{Hi}-\mathrm{C}$ measurements}

We find the chromatin interaction frequencies from the computed 3-D polymer ensembles (called simulated $\mathrm{Hi}$ C) to strongly correlate with measured $\mathrm{Hi}-\mathrm{C}$ frequencies (Fig. 2). The Pearson correlations between the simulated and measured $\mathrm{Hi}-\mathrm{C}$ frequencies have approximate mean and standard error of the mean (SEM) of $0.970 \pm 0.003$ over the 39 modeled genomic loci (see details in Additional file 1: Supplementary Information). Here, correlations were computed at $5-\mathrm{KB}$ resolution after the measured $\mathrm{Hi}-\mathrm{C}$ counts were quantile normalized according to the uniform randomly sampled polymer ensemble (Fig. 1a). This approach is motivated by similar methods for comparing gene expression microarrays [50]; it allows direct comparison between simulated ensemble frequencies and measured Hi-C counts. To exclude proximity effects owing to genomic distance, we further remove the first two diagonals from the Hi-C heatmaps; namely, all $\mathrm{Hi}-\mathrm{C}$ frequencies within $10 \mathrm{~KB}$ are excluded. The simulated and measured $\mathrm{Hi}-\mathrm{C}$ data again exhibit excellent Pearson correlations, with an approximate mean and SEM of $0.96 \pm 0.003$; more details on simulations of the 39 loci are shown in Additional file 1: Figure S3. We also computed the distance corrected Pearson correlations [51] and obtained a mean and SEM of $0.64 \pm 0.02$ (more details in Additional file 2: Table S1 and Additional file 1: Figure S4). These results indicate that our 3-D ensembles are consistent with the measured $\mathrm{Hi}-\mathrm{C}$ interaction patterns.

\section{Reconstructed single-cell chromatin structures}

We have compared our single-cell chromatin models with publicly available single-cell Dip-C data for GM12878 [15]. For each cell in the Dip-C ensemble, we identified the corresponding CHROMATIX cell with maximal overlap of contacts. Figure 3 shows the overall pattern of agreement and examples of individual single cells. In general, CHROMATIX single-cell models contain more contacts (gray regions in Fig. 3a-c) than that of Dip-C, but there is overall good agreement, with many long-range contacts appearing in both Dip-C and CHROMATIX single cells (Fig. 3a-c). The median overlap coefficient is $\sim 65 \%$ for the $n=976$ cell loci.

\section{Analysis of single-cell chromatin domains}

Motivated by single-cell optical imaging studies of Bintu et al. [52], we examined the 3-D chromatin structures at locus chrX:19,560,000-20,170,000 to assess if single-cell domains are present (Fig. 4). Our key findings are similar to that of [52], even though the cells we modeled are of different cell lineage. Specifically, diverse patterns of chromatin contacts are seen in reconstructed chromatin folds of single cells: domain-like patterns appear among singlecell distance plots (Fig. 4c), which resemble the domains in the mean distance plots (Fig. 4a). Similar to [52], there are many instances where the domain patterns are less clear. Furthermore, there is non-zero probability of forming domain boundaries at all locations of the locus, and the precise boundaries shift from cell to cell. However, we observe similarly consistent boundary strengths at similar genomic coordinates (Fig. 4b, d).

\section{3-body complexes, maximal many-body complexes, and principal loops}

For each of the 39 loci, we are interested in fully interacting 3-body complexes, which are formed by three genomic regions where the Euclidean spatial distances among all pairs of regions are $\leq 80 \mathrm{~nm}$ [47]. These 3-body complexes may be a component of a larger $(k>3)$ fully interacting complex.

We are also interested in maximal many-body complexes which are formed by $k \geq 3$ genomic regions, where all pairwise Euclidean distances are $\leq 80 \mathrm{~nm}$, and cannot be extended to include additional regions while satisfying the distance requirement. We characterize a maximal 3, 4-, 5-, or higher-order $k$-body complex by its principal 


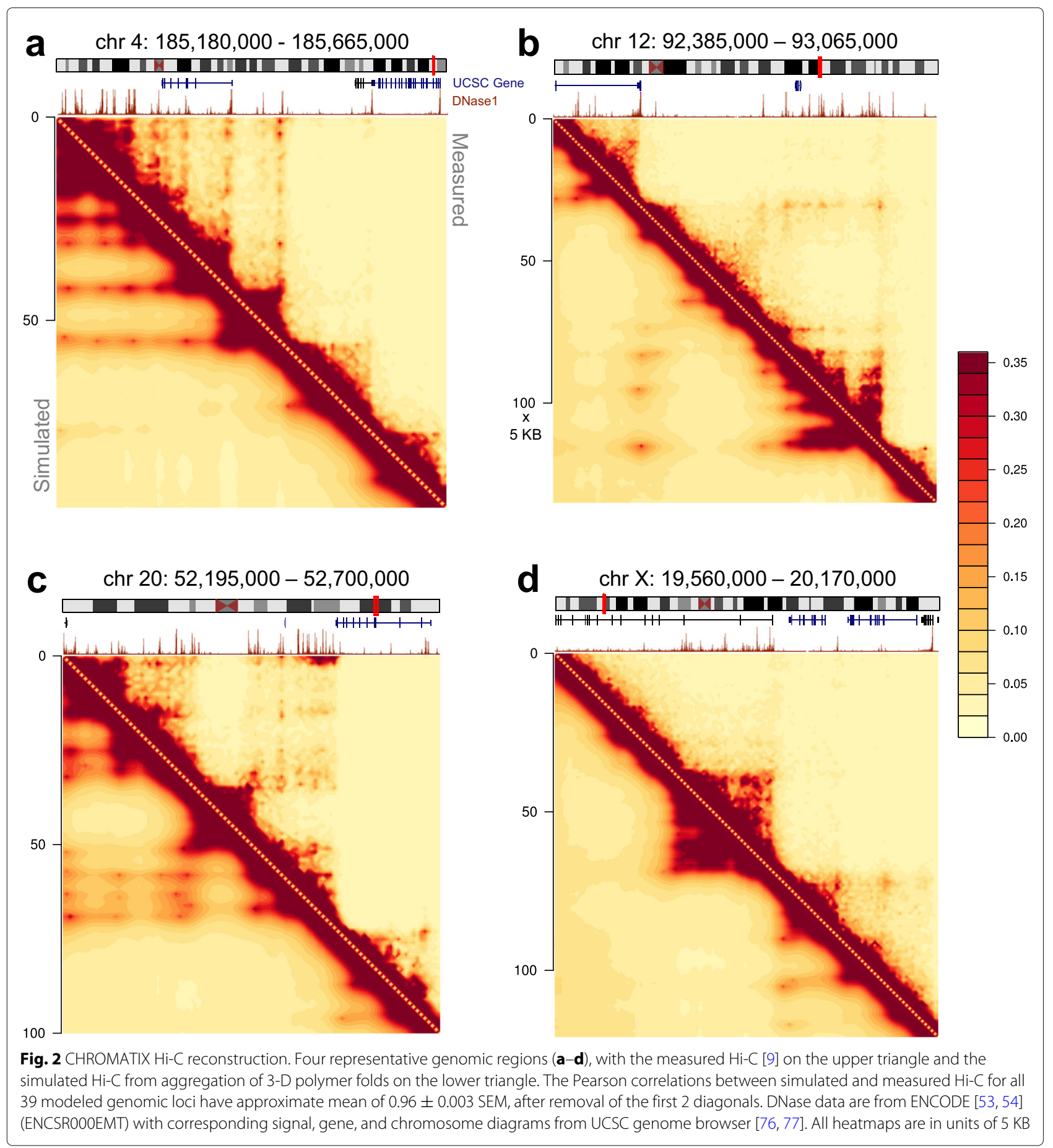

loop, which is the longest genomic span in base pairs within each $k$-body complex (Fig. 5).

Furthermore, we are interested in specific 3-body complexes and specific maximal many-body complexes, whose spatial interaction frequencies are unlikely to be observed under a uniform random folding environment (see the "Methods" section).

\section{SPRITE concordance}

We compared our predicted 3-bodies and maximal manybody principal loops, generated from population-averaged Hi-C, with publicly available SPRITE (split-pool recognition of interactions by tag extension) data for GM12878 cells [18]. The SPRITE technique captures clusters of cooccurring chromatin interactions. However, SPRITE does 


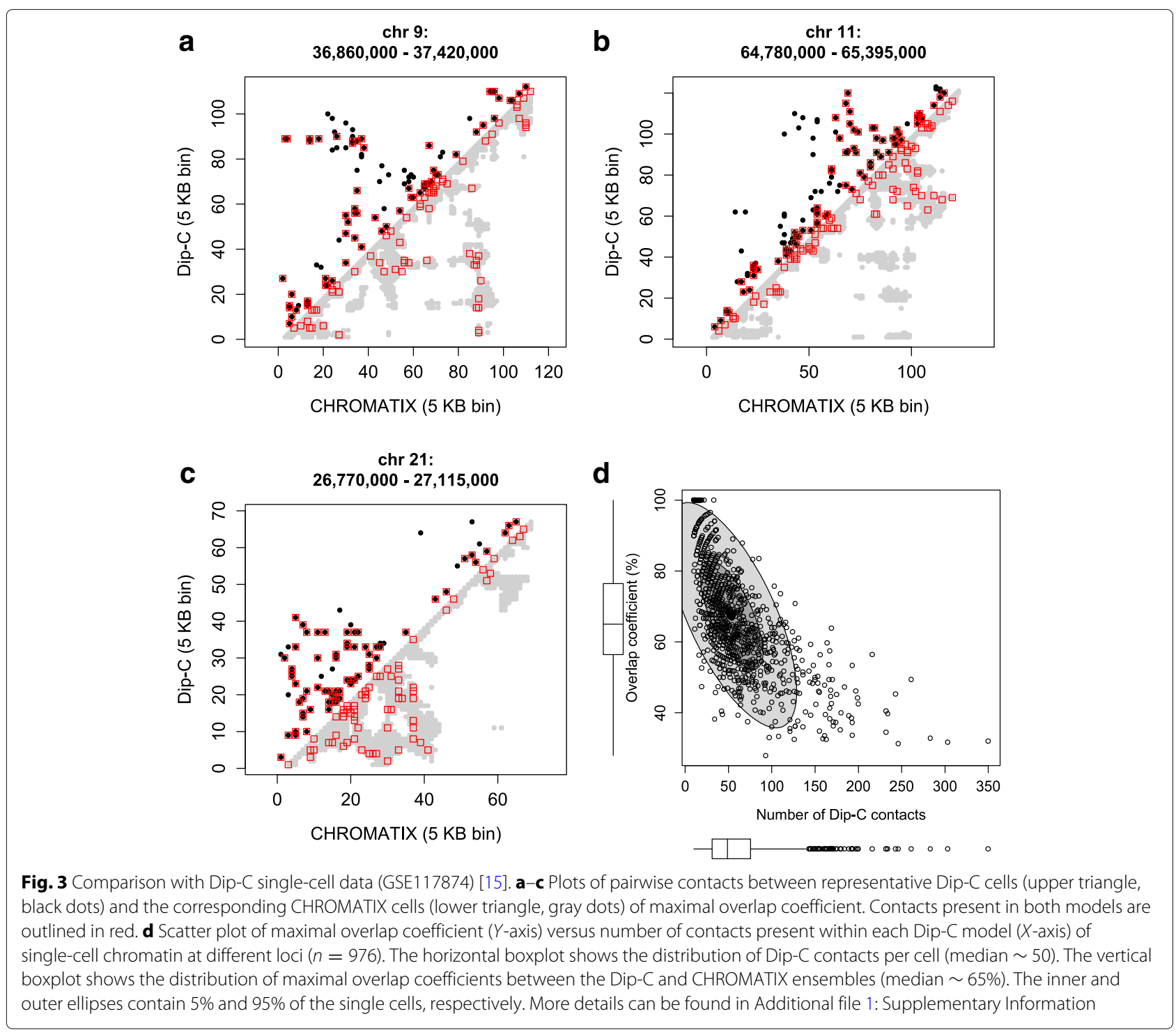

not distinguish direct from indirect cross-linking among chromatin fragments [18]-i.e., some chromatin regions present within a SPRITE cluster may not have direct spatial interactions, but, rather, may have been co-captured through a sequence of cross-links among spatially proximal regions that could extend to distances beyond the cross-linking threshold. Nevertheless, a high proportion of our predicted many-body interactions were also observed to co-occur within a SPRITE cluster; we term this proportion the found fraction. Specifically, across all 39 modeled genomic loci, we saw fairly similar median found fractions for specific and non-specific 3-bodies (approximately 90\% and 86\% respectively) as well as for principal loops (both medians approximately 99\%) at 5$\mathrm{KB}$ resolution.
To adjust for bias due to genomic distance, we stratified principal loops of many-body complexes by base pair span and computed their respective SPRITE coverage fractions, i.e., proportion of SPRITE clusters containing the principal loop. Specifically, we computed the median SPRITE coverage fraction at each $5-\mathrm{KB}$ genomic distance span for both specific and non-specific principal loops (Additional file 1: Figure S5). We found the proportion of specific median coverage fractions exceeding the corresponding non-specific coverage was significantly elevated in 29 of $39(\sim 74.4 \%)$ modeled genomic loci (FDR $<0.05$, see the "Methods" section).

We performed a similar procedure for 3-body interactions, with stratification by both principal and minor (lowest bp span) loops. In this case, the proportion of 


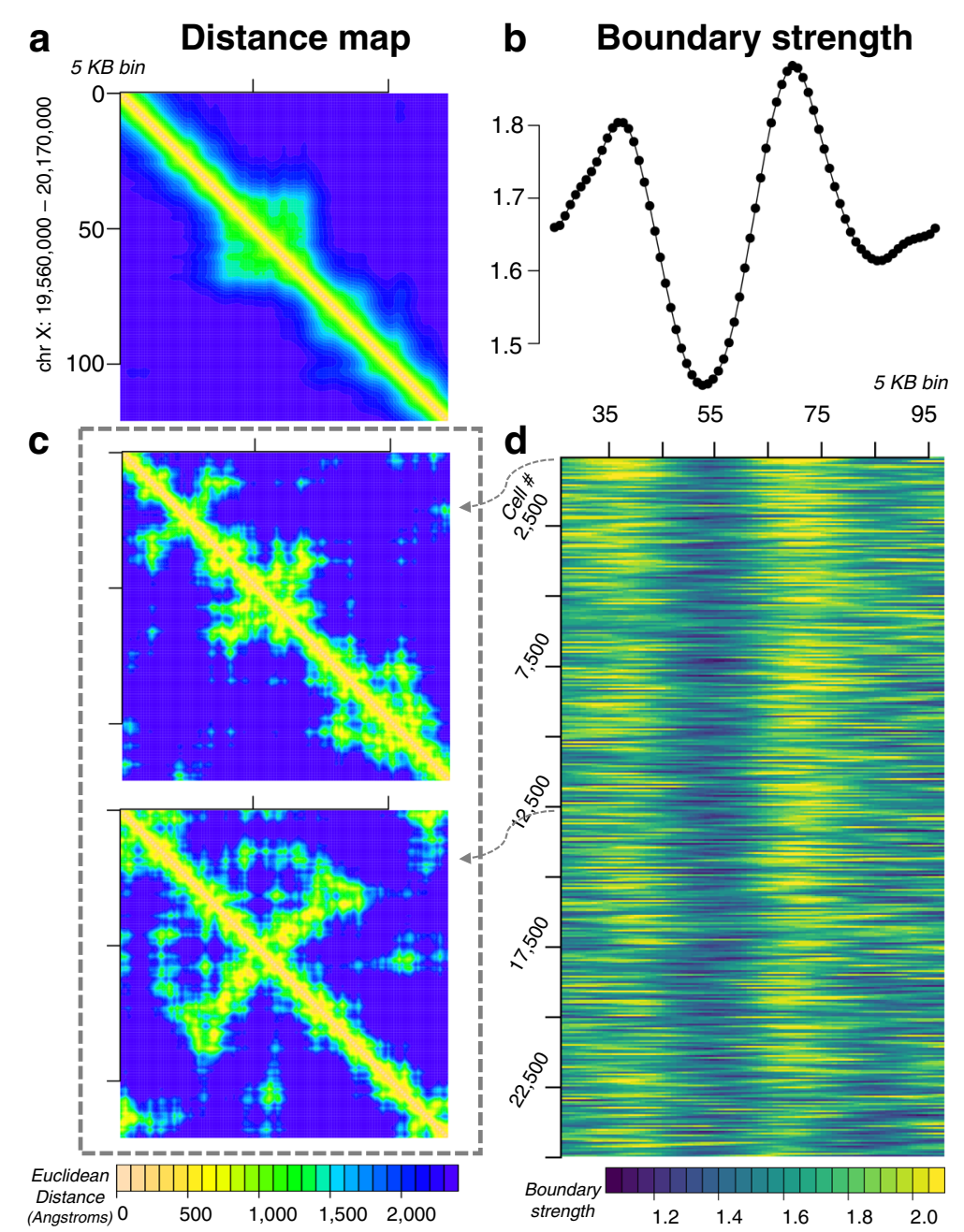

Fig. 4 Reconstructed ensemble of 25,000 single-cell chromatin structures of the locus chr X: 19,560,000 - 20,170,000 at 5-KB resolution. a Heatmap of mean pairwise Euclidean distance in $\AA$. Corresponding Hi-C heatmaps (experimental and simulated) can be seen in Fig. 2d. b Boundary strength of mean pairwise distances computed following [52] at each 5-KB bin. c Single-cell pairwise distance heatmaps for two representative cells. d Heatmap of single-cell boundary strengths, each row is the boundary strength curve of an individual cell among the 25,000 cell ensemble

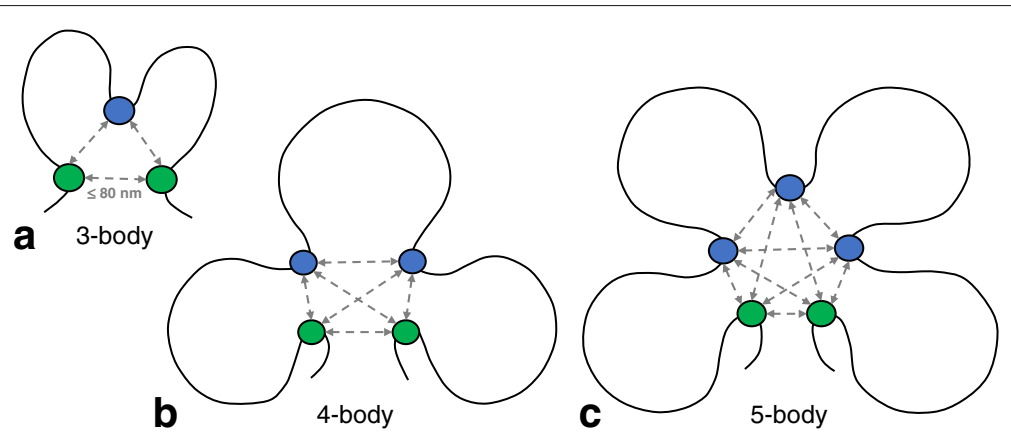

Fig. 5 Diagrams of 3-, 4-, and 5-body chromatin interactions. a-c Diagrams illustrating 3-, 4-, and 5-body chromatin interactions respectively (green and blue dots). Gray arrows represent spatial Euclidean distances within $80 \mathrm{~nm}$ [47]. The principal loop is the longest loop (in bp) among chromatin regions forming a many-body $(\geq 3)$ interaction, and genomic regions serving as anchors of principal loops are represented by green dots 
specific median coverage fractions exceeding the corresponding non-specific coverage was significantly elevated in 25 of $39(\sim 64.1 \%)$ modeled loci (FDR $<0.05$, see the "Methods" section).

Overall, we find that after controlling for genomic distance, our many-body predictions are concordant with SPRITE clusters such that specific many-bodies generally exhibit elevated SPRITE coverage over the corresponding class of non-specific many bodies. More details can be found in Additional file 1: Supplementary Information.

\section{Specific 3-body complexes are enriched in direct} interactions among functional genomic regions

Our 3-D chromatin ensembles contain rich structural information. Despite the strong effects of nuclear confinement and genomic connectivity that likely induce many bystander proximity ligations (Fig. 1a) [21, 22], our model can identify specific many-body interactions. Figure 6 provides an overview of our findings for specific 3-body interactions across the 39 super-enhancer containing loci. While functional genomic regions (i.e., super-enhancers, enhancers, and promoters) participate in both specific and non-specific 3-body interactions, the proportion of interactions with no known functional associations is markedly increased for non-specific (33 $\pm 3 \%$ SEM, Fig. 6a) compared to specific ( $19 \pm 2 \%$ SEM, Fig. $6 c)$ 3 -body interactions. Further, the medians of non-specific vs. specific 3-body interactions without functional associations (31\% and $17 \%$ respectively) are significantly different $\left(p\right.$ value $=4.5 \times 10^{-5}$ by Mann-Whitney $U$ test, Additional file 1: Figure S6a).

\section{Functional landscape of specific 3-body complexes shows interactions among super-enhancers and promoters}

The functional landscape of 3-body spatial interactions is shown in Fig. $6 \mathrm{~b}$ and d. We observe a higher proportion of specific 3-body interactions involving multiple $(\geq$ 2) super-enhancers directly co-interacting with promoters, when compared to non-specific 3-body interactions (approximately $5.5 \pm 0.6 \%$ SEM vs. $1.2 \pm 0.3 \%$ SEM respectively, with $p$ value $=1 \times 10^{-8}$ by Mann-Whitney $U$ test on the corresponding medians of $4.5 \%$ and $0.8 \%$, respectively, Additional file 1: Figure S6b). Similarly, we observe a slightly higher proportion of specific 3-body
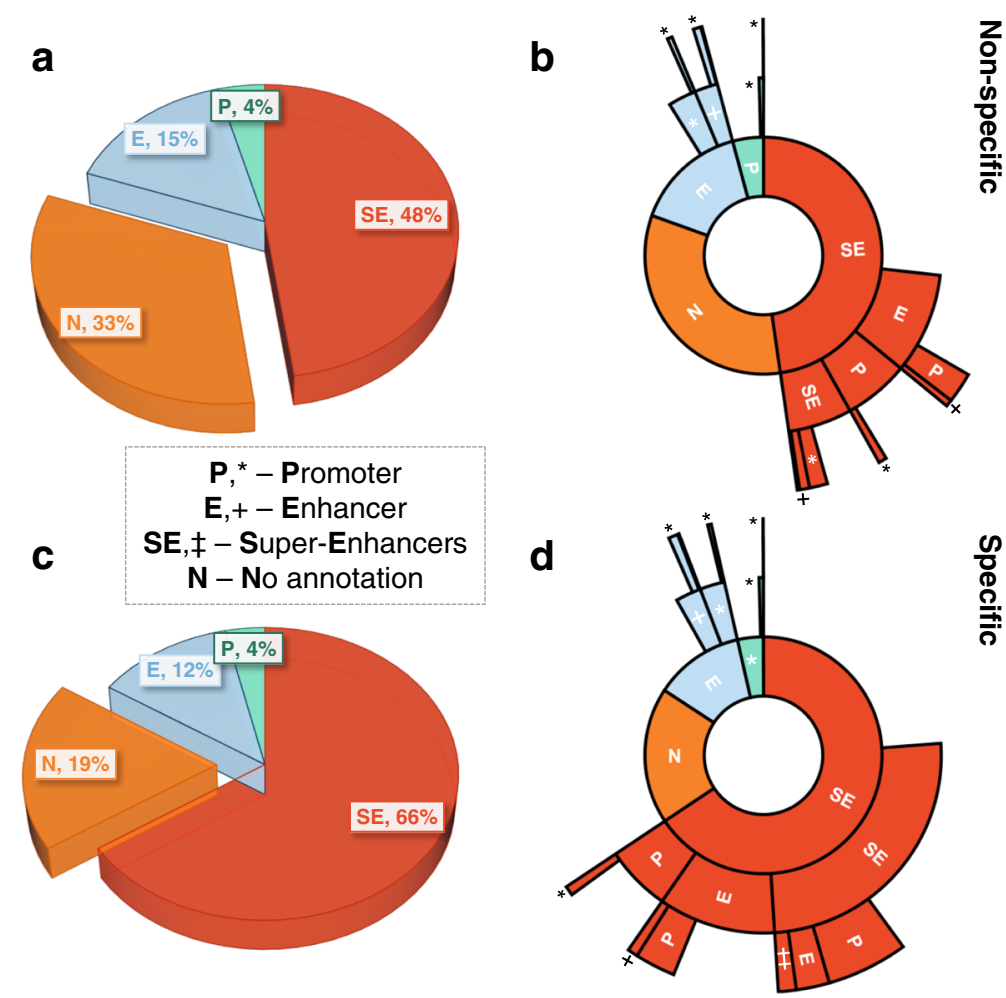

Fig. 6 Functional landscape of 3-body chromatin interactions. Pie (a, c) and corresponding sunburst (b, d) charts for the proportion of specific (bottom) and non-specific (top) 3-body interactions involving the functional genomic regions of super-enhancer (SE), enhancer (E), and promoter $(P)$. The innermost ring of the sunburst charts $(\mathbf{b}, \mathbf{d})$ are the same as the corresponding pie charts of $(\mathbf{a}, \mathbf{c})$, with outer rings representing the sub-fractions of interacting partners with $\mathrm{SE}$, E, or P functional associations. Gaps in the sunburst charts represent the fractions of interacting partners with no known $\mathrm{SE}$, E, or $\mathrm{P}$ annotation. Here, 3-body interactions are not required to be maximal and can be part of a larger many-body complex where all regions are within $80 \mathrm{~nm}$. Plots shown are the averages across all 39 modeled genomic loci 
interactions with at least 3 distinct super-enhancers relative to non-specific 3-body interactions (approximately $1.2 \pm 0.4 \%$ SEM vs. $0.2 \pm 0.1 \%$ SEM respectively at $p$ value $=$ $8.4 \times 10^{-5}$ by Mann-Whitney $U$ test on the corresponding medians of $0.5 \%$ and $0.0 \%$ respectively, Additional file 1 : Figure S6c).

\section{Functional landscape of maximal 4- and 5-body complexes} shows specific principal loops bridging super-enhancers Our high-resolution 3-D chromatin ensembles also contain information on maximal higher-order many-body interactions. Figure 7 provides an overview of the functional landscape of maximal $k$-body complexes $(k \geq 3)$ among the $39 \mathrm{SE}$-associated loci. Here a maximal $k$ body complex is defined such that it cannot be extended to form a fully interacting $k+1$ or higher complex; this is unlike the 3-body complexes depicted in Fig. 6, which may be part of still higher-order $(k \geq 4)$ fully interacting complexes. These maximal many-body complexes are grouped together by principal loop, namely, the longest genomic span in base pairs within each $k$-body interaction.

Overall, we observe an increased proportion of specific maximal 4- and 5-body complexes relative to their non-specific counterparts $(29+30=59 \pm 0.9 \%$ SEM vs. $21+19=40 \pm 0.5 \%$ SEM respectively, Fig. 7a, c). Correspondingly, we observe a markedly decreased proportion of specific maximal 3-body complexes relative to non-specific maximal 3-body complexes (12 $\pm 1 \%$ SEM and $29 \pm 1 \%$ SEM respectively, Fig. 7a, c). That is, maximal higher-order interactions beyond 3-body are preferred in the SE-associated loci.

Furthermore, we observe a higher proportion of specific principal loops bridging $\geq 2$ super-enhancers when compared to non-specific complexes, at $7.6 \pm 1.4 \%$ SEM vs. $1.9 \pm 0.5$ SEM respectively (Fig. $7 \mathrm{~b}, \mathrm{~d}$ ), with a significant $p$ value of $6.1 \times 10^{-7}$ (Mann-Whitney $U$ test on the corresponding medians of $4.1 \%$ and $0.7 \%$ respectively, Additional file 1: Figure S7a). In addition, we observe a higher proportion of specific principal loops bridging a

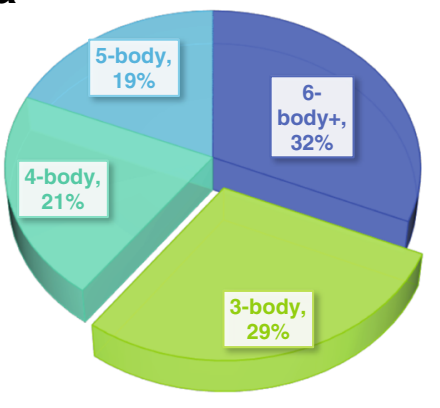

$\mathbf{P},{ }^{*}-$ Promoter; $\mathbf{E},+-$ Enhance

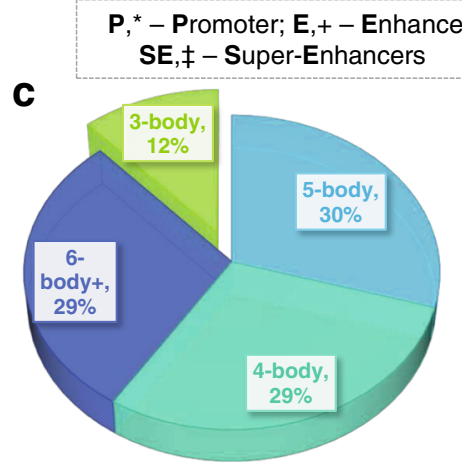

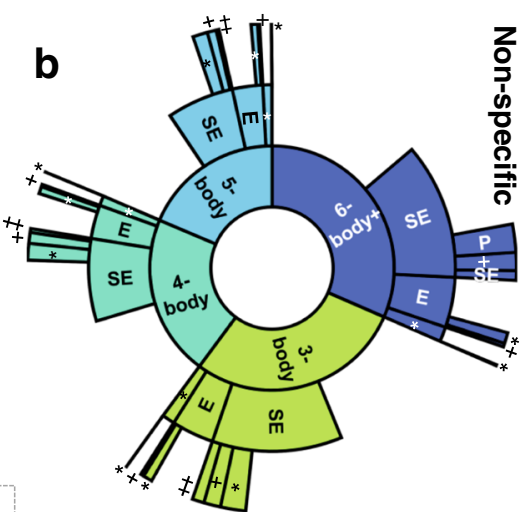

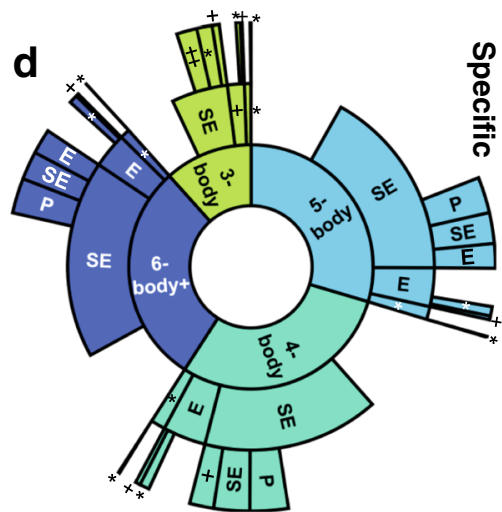

Fig. 7 Functional landscape of principal loops in many-body chromatin interactions. A principal loop is the longest loop (in bp) among chromatin regions forming a many-body $(\geq 3$ ) interaction, where all pairs of bodies (i.e., chromatin regions) forming the interaction are within $\leq 80 \mathrm{~nm}$ Euclidean distance [47]. The pie $(\mathbf{a}, \mathbf{c})$ and innermost ring of the sunburst $(\mathbf{b}, \mathbf{d})$ plots both show the proportion of specific (bottom) and non-specific (top) principal loops within maximal 3-, 4-, 5-, or $\geq 6$-body interactions; the 2 outer rings(b, d) show the corresponding fraction of principal loops with functional annotations - super-enhancer (SE), enhancer (E), promoter (P)—where gaps represent the fractions of principal loop regions with no known $\mathrm{SE}$, E, or $\mathrm{P}$ annotation. Only maximal many-body interactions are represented, i.e., no other chromatin region exists within the interaction distance such that all pairs are within $80 \mathrm{~nm}$. Plots shown are the averages across all 39 modeled genomic loci 
super-enhancers to promoters when compared to principal loops of non-specific complexes, at $8.2 \pm 0.9 \%$ SEM vs. $5.6 \pm 0.7 \%$ SEM respectively (Fig. 7b, d), with a $p$ value of 0.026 (Mann-Whitney $U$ test on the corresponding medians of $7.0 \%$ and $4.6 \%$ respectively, Additional file 1 : Figure S7b). Taken as a whole, these findings suggest that specific principal loops within higher-order complexes serve the important role of bridging functional genomic regions to allow spatial coupling.

\section{Open and transcriptionally active chromatin is predictive of regions enriched in principal loops of many-body interactions}

We then asked whether biological markers along the linear genome, such as epigenetic modifications, contained information on the specific higher-order physical interactions uncovered through our extensive 3-D modeling. While these loci with super-enhancers are enriched in active markers such as H3K27ac, we want to know if there are markers within the context of the enriched background that can differentiate regions of specific from non-specific many-body interactions. Notably, we asked whether biological markers could predict regions enriched in anchors of specific many-body principal loops.

To this end, we tested whether $5-\mathrm{KB}$ intervals enriched in specific principal loop participation could be predicted using publicly available data, e.g., the ENCODE reference epigenome for GM12878 cells (ENCSR447YYN, Additional file 3: Table S2) [53, 54]. For this task, we built a machine learning classifier based on random forest (Fig. 8, the "Methods" section) $[55,56]$.

Our predictor achieved good performance, with a mean ROC AUC of 0.804 and an out-of-bag error of $21.5 \%$ over 5 -fold cross-validation (Fig. 8c). Our results indicate that genomic intervals enriched with specific principal loop anchors can be identified by biological markers.

Inspection of our model revealed biological markers most predictive of principal loop enrichment are consistent with open chromatin and active transcriptioni.e., increased signal intensities for DNase accessibility, POLR2A binding, H3K4me1, and nuclear fraction RNA (Fig. 9). Box plots of the corresponding $z$-score signal distributions revealed significant differences among principal loop enriched versus non-enriched regions (Fig. 9b, c). The active chromatin marker H3K27ac was also significantly increased in principal loop enriched regions $(p$ value $=4.0 \times 10^{-23}$ ); however, likely due to close correlations with both DNase accessibility and H3K4me1 (Pearson coefficients of 0.81 and 0.68 respectively), H3K27ac itself was not considered as informative according to the feature importance criteria of our classifier (Fig. 9c).

We also found that chromatin architectural protein CTCF and cohesin-subunit RAD21 exhibited significantly increased ChIP-seq signal intensities in principal loop enriched regions $\left(p\right.$ value $=5.0 \times 10^{-4}$ and $7.0 \times 10^{-14}$ respectively), although RAD21 was found to be a more important predictor (Fig. 9a, c).

Consistent with increased active markers, we found decreased ChIP-seq signal intensities for the repressive mark H3K27me3 to be predictive of principal loop enrichment (Fig. 9a, c). Overall, we found open and active chromatin markers, along with decreased repressive markers, to be strongly predictive of $5-\mathrm{KB}$ intervals enriched for anchors of specific principal loops.

\section{Discussion}

We have developed a computational model for identifying specific chromatin many-body interactions and for reconstructing their functional landscapes from population $\mathrm{Hi}-$ $\mathrm{C}$ contact frequencies. Our method exploits extensive biophysical folding simulations to infer dependencies among chromatin contacts. By incorporating the inferred dependencies into a Bayesian generative model [39], our method deconvolves the intrinsic single-cell chromatin contact states underlying the pairwise, population-averaged $\mathrm{Hi}-\mathrm{C}$ data.

Our 3-D chromatin ensembles are highly realistic as they exhibit spatial interaction frequencies across many loci at Pearson correlations of $96-97 \%$ to the measured $\mathrm{Hi}-\mathrm{C}$. This close level of correlation is significant, as only basic biophysical assumptions are made (e.g., an $80-\mathrm{nm}$ interaction distance threshold and nuclear volume confinement) with no adjustable parameters. This is in contrast to several prior studies where each domain or bead modeled requires a separate adjustable parameter $[57,58]$.

Furthermore, the reconstructed 3-D chromatin ensembles are generated from a very sparse set of interactionsjust $\sim 5 \%$ of the predicted specific $\mathrm{Hi}-\mathrm{C}$ interactions are sufficient to produce polymer ensembles with contact frequencies consistent with $\mathrm{Hi}-\mathrm{C}$ measurements (Fig. 2). Notably, our models indicate that only 15-32 interactions are sufficient to reconstruct loci of size $480 \mathrm{~KB}$ to $1.94 \mathrm{MB}$. Hence, these sparsely selected sets are likely enriched with interactions driving the chromatin fold [22, 47].

Our computed 3-D chromatin ensembles contain rich structural information, allowing prediction of specific, i.e., highly non-random, many-body $(\geq 3)$ chromatin interactions. Our predictions are overall concordant with SPRITE, with a majority of modeled genomic loci exhibiting significantly elevated median coverages for specific vs. non-specific many-body interactions.

The landscape of many-body interactions emerging from our analysis of 39 active genomic loci showed super-enhancers (SE) as enriched in specific many-body principal loop participation compared to non-SE regions $\left(p=2.24 \times 10^{-129}\right.$, Additional file 1: Figure S8), with 
a

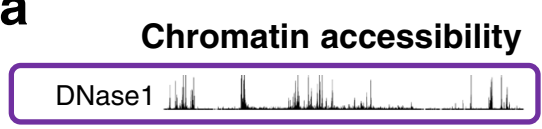

Histone marking

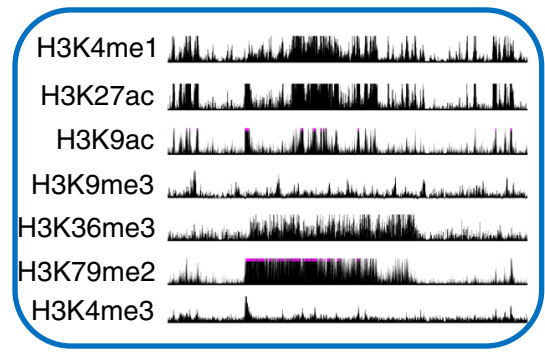

Protein binding

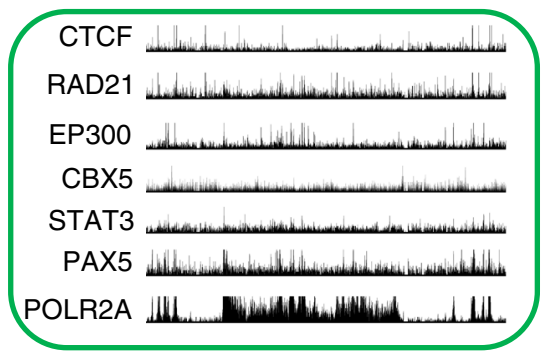

RNA expression

RNA poly(A)+
RNA poly(A)- b
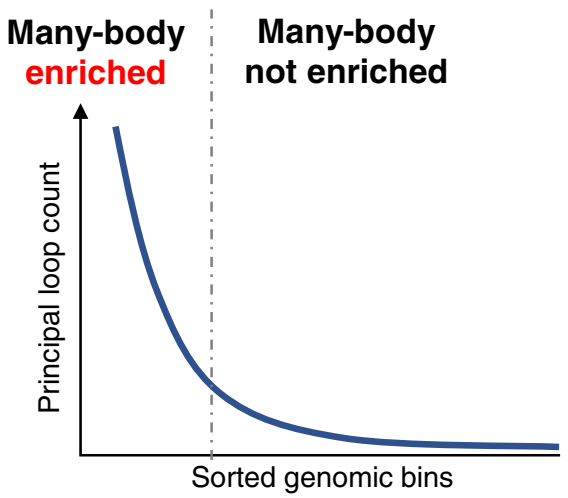

C

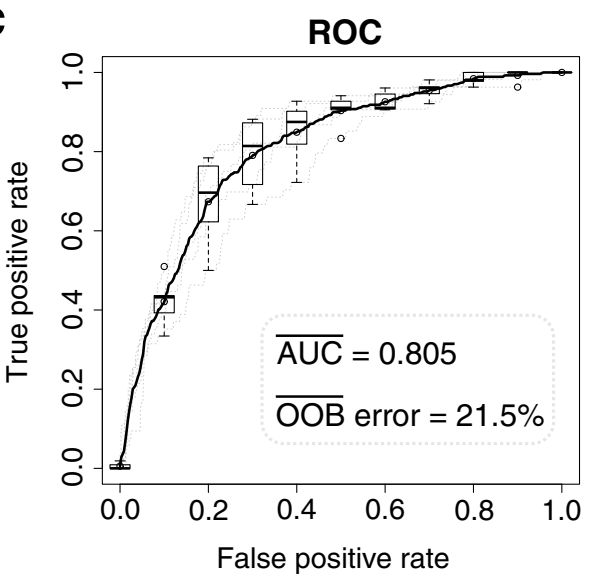

Fig. 8 Predictive model for principal loop enrichment. a Publicly available biological datasets (Additional file 3: Table S2), primarily from ENCODE reference epigenome for GM12878 (ENCSR447YYN) [53, 54], were used as predictive inputs to a random forest [55, 56] machine learning classifier. Illustrative signals shown are from the UCSC genome browser [76, 77] for locus chr 12: 11,690,000-12,210,000. b Cartoon illustration of enriched versus not enriched regions. Genomic regions, each corresponding to a non-overlapping 5-KB bin, were sorted based on principal loop participation; a subset of those occurring above the elbow inflection point were labeled as enriched; those occurring below the inflection point were labeled as not enriched (see the "Methods" section). c Receiver operating characteristic (ROC) curve [78] showing performance of our random forest classifier in discriminating principal loop enriched from not enriched genomic regions. Trained random forest model showed a mean area under the curve (AUC) of 0.805 on test set and a mean out-of-bag (OOB) error, an unbiased estimate of generalization error [55], of 21.5\% over 5-fold cross-validation

overall levels of SE-SE and SE-promoter interactions elevated in specific many-bodies (Figs. 6 and 7). While the loci studied were a priori selected based on SPRITE clusters containing multiple super-enhancers, SPRITE measurements per se cannot distinguish direct from indirect cross-linking. Therefore, to our knowledge, this work is the first to provide computational evidence, with measurable Euclidean distances estimated from our models, that super-enhancers are directly and non-randomly interacting spatially with other functional genomic regions in many-body complexes [18]. These predictions can be tested experimentally.

Our principal loop heatmaps can reveal important insight into the higher-order spatial organization of chromatin. As an example, Fig. 10 shows that at the SH3KBP1 locus, regions participating in many-body principal loops generally do not appear to be forming domains, with the exception of 3-body principal loops which appear to resemble the patterns of the original pairwise $\mathrm{Hi}$ $C$ (Fig. 2d). Instead, as evidenced by the banding patterns of the 4-, 5-, and 6-body heatmaps (bottom row of Fig. 10), principal loops may primarily be facilitating direct, long-range interactions among functional genomic regions such as super-enhancers, enhancers, and promoters. Such banding patterns at $5 \mathrm{~KB}$ are likely not due to A/B compartmentalization (100 KB-1 MB scale), as our loci are mostly (>90\%, Additional file 2: Table S1) in A compartments. This is consistent with our functional landscapes exhibiting decreased preference for maximal 3-body complexes and relatively increased functional associations among specific many-bodies (Figs. 6 and 7). 


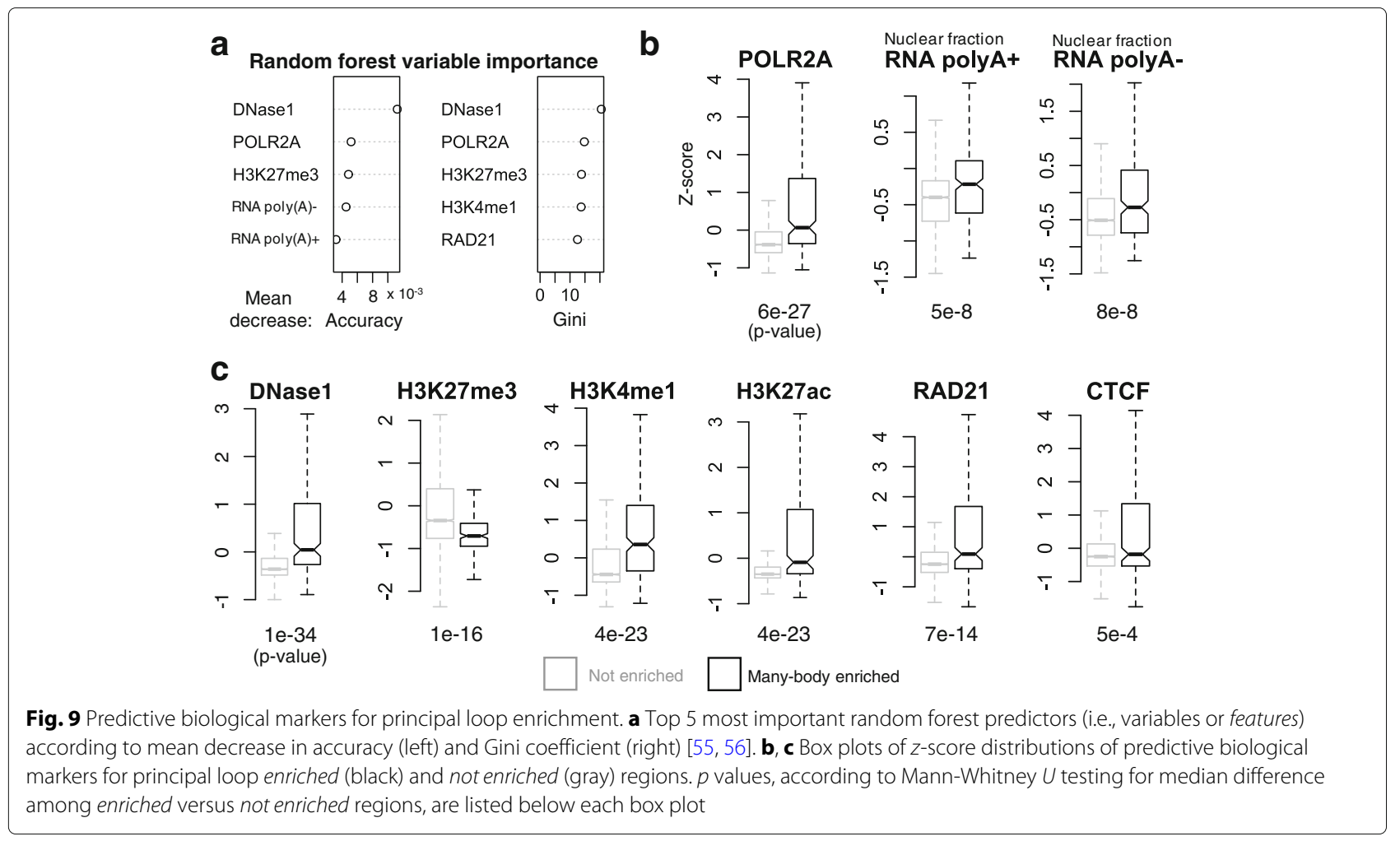

In contrast to other models which focus on heterochromatin condensation [29], we instead examine highly active chromatin regions. Our analysis showed that even in super-enhancer loci where active markers are enriched at baseline, open chromatin (DNase hypersensitivty) and the presence of active transcriptional marks such as
POLR2A and nuclear fraction RNA are predictive of 5-KB regions enriched for anchors of specific many-body principal loops. Our findings are consistent with the opinion that nuclear RNAs may be important factors for nuclear organization through promotion of phase separation and ultimately enhancer-promoter looping [59, 60].

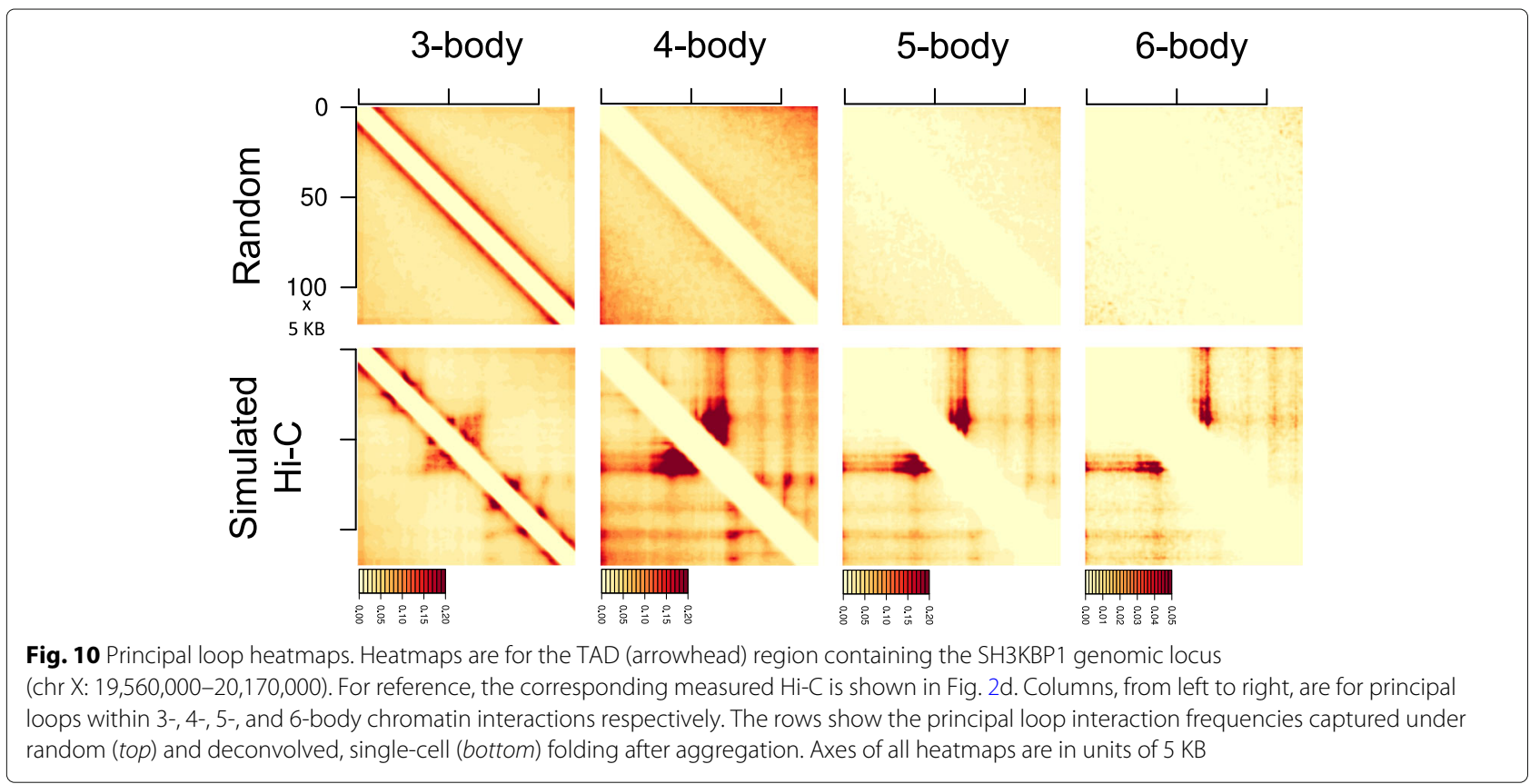




\section{Conclusions}

We have developed CHROMATIX, a computational framework for predicting the intrinsic 3-D structural ensembles underlying population-averaged $\mathrm{Hi}-\mathrm{C}$ data; our method is general and can be applied to other cell lines where pairwise chromatin contact information is available. We demonstrate our predicted 3-D structural ensembles have close correlation with the measured $\mathrm{Hi}$ $\mathrm{C}$ data over 39 modeled genomic loci. Our CHROMATIX framework can also identify specific many-body chromatin interactions, and we show the predicted manybody interactions to be broadly concordant with SPRITE clusters.

We find our predicted specific many-body interactions to be significantly associated with functional genomic regions such as SEs and promoters; further, they preferentially form maximal 4- or higher-order interactions over 3-body interactions. These findings are consistent with specific principal loops likely playing the important role of bridging many genomically distant regions and allowing them to condense into functional assemblies through direct spatial contact. Overall, the many-body interactions uncovered in this study may serve as the 3-D manifestations of phase-separated, multi-valent assemblies among super-enhancer regions [10].

Further, we have shown that genomic regions enriched in anchors of principal loops are also enriched in open and active chromatin marks, such as DNase accessibility, POLR2A, H3K4me1, H3K27ac, and nuclear fraction RNA, and depleted in the repressive mark H3K27me3. These biological markers are likely representative of factors needed to condense distant chromatin regions into ordered, spatial complexes necessary to regulate fundamental cellular processes such as gene transcription.

The CHROMATIX method has the promise of generating high-resolution 3-D ensembles of chromatin structures with detailed information of spatial manybody interactions using abundantly available populationaveraged $\mathrm{Hi}-\mathrm{C}$ data. As only about $5 \%$ of specific interactions are sufficient to reproduce measured $\mathrm{Hi}-\mathrm{C}$ frequencies, CHROMATIX can provide higher resolution details beyond that of input Hi-C measurement.

Our method enables quantification of the extent of specific 3-, 4-, and higher-order many-body interactions at a large scale. It also elucidates the functional implications by providing details on how super-enhancers, enhancers, promoters, and other functional units probabilistically assemble into a spatial apparatus with measurable Euclidean distances. Our method can predict specific many-body interactions solely from markers along the linear genome and allows insight into the biological factors that drive the spatial coordination among genomic regions. Finally, our method can simulate multiple independent loci located on separate chromosomes within the same confining nuclear volume and can be applied to identify specific inter-chromosomal manybody interactions.

\section{Methods}

We now provide technical details on key components of the CHROMATIX method (Fig. 1).

\section{Calculating $p$ values for calling specific $\mathrm{Hi}-\mathrm{C}$ interactions}

To assign statistical significance $p$ values to each $\mathrm{Hi}$ $C$ measured interaction, we use a scalable Bag of Little Bootstraps resampling procedure [61] over the uniform random 3-D polymer ensemble, with 10,000 outer replicates, to obtain a null distribution over random chromatin contacts. $p$ values are assigned to each $\mathrm{Hi}-\mathrm{C}$ contact frequency based on the proportion of bootstrap replicate contact frequencies exceeding the measured $\mathrm{Hi}-\mathrm{C}$ at the same genomic distance.

\section{Polymer simulation of structural perturbations}

To predict which specific contacts are likely co-occurring within individual cells of the population, we carried out extensive structural perturbation simulations. These biophysical simulations were used to elucidate dependencies and infeasible geometries among chromatin contacts. We incorporated information from the perturbed simulations into a sparsity-inducing Bayesian prior distribution over hypothetical folding mechanisms among the specific contacts, where each mechanism is encoded in the form of a directed acyclic graph (DAG) [62, 63]. A considered DAG, in which each edge represents a possible causal dependency between two contacts, is restricted according to computational knock-in perturbations supporting such a hypothesis; specifically, if knocking-in a contact is observed to significantly upregulate the frequency of another contact beyond random, a directed edge from the knocked-in contact to the upregulated contact is then available to be sampled when generating folding mechanisms. Given the observed population $\mathrm{Hi}-\mathrm{C}$ data and the results of simulated biophysical perturbations, we infer the posterior distribution of single-cell contact states through Gibbs sampling (see Additional file 1: Supplementary Information for details on sampling procedures). We find that our models for 38 out of the 39 loci have higher posterior probabilities than the naive models of product of independent pairwise contacts. The naive models further suffer from the inability to recognize geometrically infeasible combinations of pairwise contacts.

\section{Functional annotation and loci selection}

We used LILY [64] to detect functional genomic regions containing super-enhancers, enhancers, and promoters based on H3K27ac ChIP-seq data of GM12878 cells [65](see Additional file 4: Table S3). We used publicly 
available SPRITE data for GM12878 cells [18] to select clusters containing multiple $(\geq 2)$ super-enhancers as a basis for investigating if many-body interactions may form among multiple super-enhancers. We then used publicly available $\mathrm{Hi}-\mathrm{C}$ data for GM12878 at 5-KB resolution [9] to identify the median TAD ( $\leq 2 \mathrm{MB}$, arrowhead domain) boundaries for the considered SPRITE clusters. After discarding regions with greater than $\sim 25 \%$ overlap, we obtained 39 genomic loci (Additional file 2: Table S1), 35 of which have no overlap, for further investigation of many-body interactions. Hi-C contact counts at each locus, normalized via Knight-Ruiz matrix balancing [66], were obtained using Juicer [67] also at 5-KB resolution.

\section{Cliques and maximal many-body interactions}

We extend the nCSAC approach of Gürsoy et al. [21, 22] to identify specific many-body $(\geq 3$ ) chromatin interactions. We define a many-body interaction as a complex of 5 -KB chromatin regions such that the Euclidean distances between all pairs of regions in the complex are within a cross-linking threshold of $\leq 80 \mathrm{~nm}$ [47]. Using graph theory terminology, a many-body interaction is equivalent to a clique [68], i.e., a fully connected graph such that all pairs of vertices are connected by undirected edges. Further, a many-body complex, or clique, is maximal if no additional chromatin regions may be added such that all pairs remain within the cross-linking threshold. We use the highly optimized graph analysis library igraph to detect many-body interactions within a 3-D polymer [69].

\section{Calling specific many-body interactions}

To generate a null distribution over many-body chromatin interactions, we first tally the frequency of each observed many-body interaction within a uniform randomly folded ensemble of 75,000 polymers. We repeat the tally procedure by bootstrap resampling over the full polymer ensemble for 1000 total replicates; this produces a distribution over the many-body interaction frequencies under a null hypothesis of random folding. For 3-body interactions (Fig. 6), we detect all cliques consisting of exactly 3 distinct chromatin regions and do not require them to be maximal; that is, these 3-bodies may be part of a larger fully connected complex. For principal loop analysis, we detect cliques consisting of at least 3 distinct chromatin regions and require that each clique is maximal (Fig. 7).

We then identify specific many-body interactions at a locus by first tallying the corresponding many-body frequencies within each sample of the CHROMATIX deconvolved $\mathrm{Hi}-\mathrm{C}$ ensemble (i.e., simulated $\mathrm{Hi}-\mathrm{C}$ ) of 25,000 polymers. We stratify the many-body frequencies (random and simulated $\mathrm{Hi}-\mathrm{C}$ ) according to both genomic distance and clique size. Specifically, for 3-body interactions shown in Fig. 6, we stratify all frequencies based on principal (i.e., longest) and minor (i.e., shortest) loop spans in base pairs. For maximal principal loop interactions shown in Fig. 7, we stratify based on clique size and the base pair span of the principal loop. Stratification is necessary to control for genomic distance bias, i.e., the fact that genomic regions with short genomic separation tend to spatially co-locate [21] and that larger clique sizes tend to allow correspondingly longer genomic distances to interact spatially with increased frequency. We assign a $p$ value to each simulated $\mathrm{Hi}-\mathrm{C}$ many-body frequency as the within-stratum proportion of random (bootstrapreplicated) many-body frequencies that exceed the simulated Hi-C many-body frequency. Finally, to control for multiple testing, a simulated $\mathrm{Hi}-\mathrm{C}$ many-body interaction is called specific if the FDR-adjusted [70] $p$ value is $<0.05$.

\section{Concordance with sPRITE}

We compared our 3-body and maximal many-body principal loop predictions with publicly available SPRITE data for GM12878 [18]. To adjust for genomic distance bias, we stratified principal loops according to base pair span and computed the SPRITE coverage fraction, i.e., proportion of SPRITE clusters that contained each principal loop complex. Specifically, we computed the median SPRITE coverage fraction at each $5-\mathrm{KB}$ genomic distance span for both specific and non-specific principal loops (Additional file 1: Figure S5). At each of the 39 modeled loci, we assessed the significance of the proportion of specific medians exceeding the corresponding non-specific medians by permutation testing: we randomly permuted the specific and non-specific labels assigned to each principal loop and re-computed the proportion of specific medians exceeding non-specific medians for 1000 total replicates. We then assigned a $p$ value to each locus by the fraction of permutation replicates exceeding the observed proportion. A similar procedure was performed for 3body predictions, with stratification by both principal and minor loop. To control for multiple testing, $p$ values where called significant if $<0.05$ after FDR correction [70].

\section{Predictive model for principal loop enrichment}

We built a random forest machine learning classifier [55] to identify biological markers predictive of regions enriched in the principal loop anchors of many-body complexes. We used publicly available biological datasets (Additional file 3: Table S2), primarily from ENCODE reference epigenome for GM12878 (ENCSR447YYN) [53, 54], as our input features (Fig. 8a). At each of the 39 modeled loci, genomic regions corresponding to nonoverlapping $5-\mathrm{KB}$ bins were sorted based on principal loop participation; a subset of those occurring above the "elbow" inflection point (Fig. 8b) were labeled as enriched; those occurring below the inflection point were labeled as not enriched. To avoid ambiguous labels and to provide a more robust decision boundary among enriched 
versus not enriched regions, we retained the top 20\% of the above-elbow fraction at each locus and discarded the remainder, while still retaining all samples below the elbow. Our final data set consisted of 231 regions enriched (i.e., positive) in many-body interactions and 5800 regions not-enriched (i.e., negative). To control for potential class imbalance issues during training, we used the randomForest $\mathrm{R}$ package [56] with stratified resampling to present equal number of positive and negative samples to each decision tree $(n=500)$ in the random forest. Classifier performance results, mean ROC AUC of 0.805 and out-of-bag error of $21.5 \%$ (Fig. 8c), were obtained on a held out test set ( $\sim 20 \%$ of labeled samples) over 5 -fold cross-validation using the caret $\mathrm{R}$ package [71].

\section{Supplementary information}

Supplementary information accompanies this paper at https://doi.org/10.1186/s13059-019-1904-z.

Additional file 1: Supplementary information. Detailed methodology for fractal Monte Carlo sampling and Bayesian deconvolution of $\mathrm{Hi}-\mathrm{C}$ specific contacts. Also, additional figures referenced for this study.

Additional file 2: Table S1. Table of genomic regions modeled for this study.

Additional file 3: Table S2. Table of public biological datasets used for predictive modeling of genomic regions enriched in many-body principal loop participation.

Additional file 4: Table S3. Table of LILY [64] annotations for super-enhancer, enhancer, and promoter genomic regions.

Additional file 5: Review history.

\section{Acknowledgements}

We thank Daniel M. Czajkowsky and Gamze Gürsoy for helpful discussions, Lin Du for the help with A/B compartment calling, and Kimberly Beiting for her editing. This work is supported by grants NIH R21Al126308, R21Al137865, R01CA204962, and R35GM127084 (to JL) and CAS-18H100000104 and NSFC-81627801 (to ZFS).

\section{Review history}

The review history is available as Additional file 5 .

\section{Authors' contributions}

APR and $J$ conceived and designed the study. APR designed and implemented the algorithms. APR carried out computation and analysis, with assistance from QS. BW assisted in computing. VB provided tool and analysis of genome annotations. ZS provided additional analysis and contributed resources. JL supervised the overall study. APR and JL wrote the paper with input from QS, VB, and ZS and assistance from BW. All authors read and approved the final manuscript.

\section{Availability of data and materials}

$\mathrm{C}++$ source code for chromatin polymer folding as well as comprehensive tutorials demonstrating how to configure polymer folding simulations are publicly available via git repository [72]. Similarly, C++ source code for our Bayesian $\mathrm{Hi}-\mathrm{C}$ deconvolution Gibbs sampler and loci modeling scripts are also available via git $[73,74]$. The source code versions used specifically in this work are available via Zenodo open-access repository [75]. All source code is licensed under the GNU General Public License v3.0.

\section{Ethics approval and consent to participate}

Not applicable.

\section{Competing interests}

The authors declare that they have no competing interests.

\section{Author details}

${ }^{1}$ Richard and Loan Hill Department of Bioengineering, University of Illinois at Chicago, Chicago, IL, USA. ${ }^{2}$ Shanghai Center for Systems Biomedicine, Shanghai Jiao Tong University, Shanghai, China. ${ }^{3}$ Institut Cochin, INSERM U1016, CNRS UMR 8104, Paris Descartes University UMR-S1016, 75014 Paris, France. ${ }^{4}$ Department of Computer Science, ETH Zurich, Zürich, Switzerland. ${ }^{5}$ State Key Laboratory for Oncogenes and Bio-ID Center, School of Biomedical Engineering, Shanghai Jiao Tong University, Shanghai, China.

Received: 4 August 2019 Accepted: 27 November 2019

Published online: 16 January 2020

\section{References}

1. Weintraub AS, Li CH, Zamudio AV, Sigova AA, Hannett NM, Day DS, Abraham BJ, Cohen MA, Nabet B, Buckley DL, et al. Yy1 is a structural regulator of enhancer-promoter loops. Cell. 2017;171(7):1573-88.

2. Oudelaar AM, Davies JOJ, Hanssen LLP, Telenius JM, Schwessinger R, Liu Y, Brown JM, Downes DJ, Chiariello AM, Bianco S, Nicodemi M, Buckle VJ, Dekker J, Higgs DR, Hughes JR. Single-allele chromatin interactions identify regulatory hubs in dynamic compartmentalized domains. 2018;50(12):1744-1751. http://doi.org/10.1038/s41588-018-0253-2.

3. Whyte WA, Orlando DA, Hnisz D, Abraham BJ, Lin CY, Kagey MH, Rahl $P B$, Lee TI, Young RA. Master transcription factors and mediator establish super-enhancers at key cell identity genes. Cell. 2013;153(2):307-19.

4. Hnisz D, Abraham BJ, Lee TI, Lau A, Saint-André V, Sigova AA, Hoke HA Young RA. Super-enhancers in the control of cell identity and disease. Cell. 2013;155(4):934-47.

5. Dekker J, Rippe K, Dekker M, Kleckner N. Capturing chromosome conformation. Science. 2002;295(5558):1306-11.

6. Lieberman-Aiden E, Van Berkum NL, Williams L, Imakaev M, Ragoczy T, Telling A, Amit I, Lajoie BR, Sabo PJ, Dorschner MO, et al. Comprehensive mapping of long-range interactions reveals folding principles of the human genome. Science. 2009;326(5950):289-93.

7. Zhang Y, McCord RP, Ho YJ, Lajoie BR, Hildebrand DG, Simon AC, Becker MS, Alt FW, Dekker J. Spatial organization of the mouse genome and its role in recurrent chromosomal translocations. Cell. 2012;148(5):908-21.

8. Dixon JR, Jung I, Selvaraj S, Shen $Y$, Antosiewicz-Bourget JE, Lee AY, Ye Z, Kim A, Rajagopal N, Xie W, Diao Y, Liang J, Zhao H, Lobanenkov W, Ecker JR, Thomson JA, Ren B. Chromatin architecture reorganization during stem cell differentiation. Nature. 2015;518(7539):331-6.

9. Rao SS, Huntley MH, Durand NC, Stamenova EK, Bochkov ID, Robinson JT, Sanborn AL, Machol I, Omer AD, Lander ES, et al. A 3D map of the human genome at kilobase resolution reveals principles of chromatin looping. Cell. 2014;159(7):1665-80.

10. Hnisz D, Shrinivas K, Young RA, Chakraborty AK, Sharp PA. A phase separation model for transcriptional control. Cell. 2017;169(1):13-23.

11. Sabari BR, Dall'Agnese A, Boija A, Klein IA, Coffey EL, Shrinivas K, Abraham BJ, Hannett NM, Zamudio AV, Manteiga JC, Li CH, Guo YE, Day DS, Schuijers J, Vasile E, Malik S, Hnisz D, Lee TI, Cisse II, Roeder RG Sharp PA, Chakraborty AK, Young RA. Coactivator condensation at super-enhancers links phase separation and gene control. Science. 2018;361(6400):. https://doi.org/10.1126/science.aar3958. https://science. sciencemag.org/content/361/6400/eaar3958.full.pdf.

12. Nagano T, Lubling Y, Stevens TJ, Schoenfelder S, Yaffe E, Dean W, Laue ED, Tanay A, Fraser P. Single-cell hi-c reveals cell-to-cell variability in chromosome structure. Nature. 2013;502(7469):59.

13. Ramani V, Deng X, Qiu R, Gunderson KL, Steemers FJ, Disteche CM, Noble WS, Duan Z, Shendure J. Massively multiplex single-cell Hi-C. Nat Methods. 2017;14(3):263.

14. Stevens TJ, Lando D, Basu S, Atkinson LP, Cao Y, Lee SF, Leeb M, Wohlfahrt KJ, Boucher W, O'Shaughnessy-Kirwan A, et al. 3D structures of individual mammalian genomes studied by single-cell Hi-C. Nature. 2017:544(7648):59.

15. Tan L, Xing D, Chang C-H, Li H, Xie XS. Three-dimensional genome structures of single diploid human cells. Science. 2018;361(6405):924-8

16. Tan L, Xing D, Daley N, Xie XS. Three-dimensional genome structures of single sensory neurons in mouse visual and olfactory systems. Nat Struct Mol Biol. 2019;26(4):297-307. https://doi.org/10.1038/s41594-019-0205-2.

17. Beagrie RA, Scialdone A, Schueler M, Kraemer DC, Chotalia M, Xie SQ, Barbieri M, de Santiago I, Lavitas L-M, Branco MR, et al. Complex 
multi-enhancer contacts captured by genome architecture mapping. Nature. 2017;543(7646):519.

18. Quinodoz SA, Ollikainen N, Tabak B, Palla A, Schmidt JM, Detmar E, Lai MM, Shishkin AA, Bhat $P$, Takei Y, et al. Higher-order inter-chromosomal hubs shape 3D genome organization in the nucleus. Cell. 2018;174(3): 744-57.

19. Bickhart DM, Rosen BD, Koren S, Sayre BL, Hastie AR, Chan S, Lee J, Lam ET, Liachko I, Sullivan ST, et al. Single-molecule sequencing and chromatin conformation capture enable de novo reference assembly of the domestic goat genome. Nat Genet. 2017;49(4):643.

20. Schmitt AD, Hu M, Ren B. Genome-wide mapping and analysis of chromosome architecture. Nat Rev Mol Cell Biol. 2016;17(12):743.

21. Gürsoy G, Xu Y, Kenter AL, Liang J. Spatial confinement is a major determinant of the folding landscape of human chromosomes. Nucleic Acids Res. 2014;42(13):8223-30.

22. Gürsoy G, Xu Y, Kenter AL, Liang J. Computational construction of 3D chromatin ensembles and prediction of functional interactions of alpha-globin locus from 5C data. Nucleic Acids Res. 2017;45(20):11547-58.

23. Gürsoy G, Xu Y, Liang J. Spatial organization of the budding yeast genome in the cell nucleus and identification of specific chromatin interactions from multi-chromosome constrained chromatin model. PLoS Comput Biol. 2017;13(7):1005658.

24. Barbieri M, Chotalia M, Fraser J, Lavitas L-M, Dostie J, Pombo A, Nicodemi M. Complexity of chromatin folding is captured by the strings and binders switch model. Proc Natl Acad Sci. 2012;109(40):16173-8.

25. Kinney NA, Sharakhov IV, Onufriev AV. Investigation of the chromosome regions with significant affinity for the nuclear envelope in fruit fly-a model based approach. PLoS ONE. 2014;9(3):91943.

26. Di Pierro M, Zhang B, Aiden EL, Wolynes PG, Onuchic JN. Transferable model for chromosome architecture. Proc Natl Acad Sci. 2016;113(43): 12168-73.

27. Zhang B, Wolynes PG. Genomic Energy Landscapes. Biophys J. 2017;112(3):427-33.

28. Di Pierro M, Cheng RR, Lieberman Aiden E, Wolynes PG, Onuchic JN. De novo prediction of human chromosome structures: epigenetic marking patterns encode genome architecture. Proc Natl Acad Sci USA. 2017;114(46):12126-31.

29. MacPherson Q, Beltran B, Spakowitz AJ. Bottom-up modeling of chromatin segregation due to epigenetic modifications. Proc Natl Acad Sci. 2018;115(50):12739-44.

30. Esposito A, Annunziatella C, Bianco S, Chiariello AM, Fiorillo L, Nicodemi M. Models of polymer physics for the architecture of the cell nucleus. WIREs Syst Biol Med. 2019;11(4):1444. https://doi.org/10.1002/wsbm.1444.

31. Kinney NA, Sharakhov IV, Onufriev AV. Chromosome-nuclear envelope attachments affect interphase chromosome territories and entanglement. Epigenet Chromatin. 2018;11(1):3.

32. Shi G, Liu L, Hyeon C, Thirumalai D. Interphase human chromosome exhibits out of equilibrium glassy dynamics. Nat Commun. 2018;9(1):3161.

33. Liu L, Shi G, Thirumalai D, Hyeon C. Chain organization of human interphase chromosome determines the spatiotemporal dynamics of chromatin loci. PLoS Comput Biol. 2018;14(12):1006617.

34. Qi Y, Zhang B. Predicting three-dimensional genome organization with chromatin states. PLoS Comput. Biol. 2019;15(6):1007024.

35. Jin F, Li Y, Dixon JR, Selvaraj S, Ye Z, Lee AY, Yen C-A, Schmitt AD, Espinoza CA, Ren B. A high-resolution map of the three-dimensional chromatin interactome in human cells. Nature. 2013;503(7475):290.

36. Ay F, Bailey TL, Noble WS. Statistical confidence estimation for Hi-C data reveals regulatory chromatin contacts. Genome Res. 2014;24(6):999-1011.

37. Mifsud B, Martincorena I, Darbo E, Sugar R, Schoenfelder S, Fraser P, Luscombe NM. Gothic, a probabilistic model to resolve complex biases and to identify real interactions in Hi-C data. PloS ONE. 2017;12(4): 0174744.

38. Xu Z, Zhang G, Jin F, Chen M, Furey TS, Sullivan PF, Qin Z, Hu M, Li Y. A hidden markov random field-based bayesian method for the detection of long-range chromosomal interactions in $\mathrm{Hi}-\mathrm{C}$ data. Bioinformatics. 2015;32(5):650-6.

39. Bishop CM. Pattern recognition and machine learning (Information Science and Statistics). Berlin, Heidelberg: Springer; 2006.

40. Nora EP, Lajoie BR, SchulzEG, Giorgetti L, Okamoto I, Servant N, Piolot T, van Berkum NL, Meisig J, Sedat J, et al. Spatial partitioning of the regulatory landscape of the $x$-inactivation centre. Nature. 2012;485(7398): 381.

41. Dixon JR, Selvaraj S, Yue F, Kim A, Li Y, Shen Y, Hu M, Liu JS, Ren B. Topological domains in mammalian genomes identified by analysis of chromatin interactions. Nature. 2012;485(7398):376.

42. In: Rippe K, editor. Genome organization and function in the cell nucleus. Weinheim: WILEY-VCH Verlag GmbH \& Co. KGaA; 2012.

43. Van Holde KE. Chromatin. New York: Springer; 1989.

44. Sanborn AL, Rao SS, Huang S-C, Durand NC, Huntley MH, Jewett Al, Bochkov ID, Chinnappan D, Cutkosky A, Li J, et al. Chromatin extrusion explains key features of loop and domain formation in wild-type and engineered genomes. Proc Natl Acad Sci. 2015;112(47):6456-65.

45. Perez-Rathke A, Mali S, Du L, Liang J. Alterations in chromatin folding patterns in cancer variant-enriched loci. In: 2019 IEEE EMBS International Conference on Biomedical \& Health Informatics (BHI). IEEE; 2019. p. 1-4. https://doi.org/10.1109/BHI.2019.8834565.

46. Sun Q, Perez-Rathke A, Czajkowsky DM, Shao Z, Liang J. High-resolution single-cell models of ensemble chromatin structures during Drosophila embryogenesis from population Hi-C. bioRxiv. 2019. https://doi.org/10. $1101 / 854257$.

https://www.biorxiv.org/content/early/2019/11/25/854257.full.pdf.

47. Giorgetti L, Galupa R, Nora EP, Piolot T, Lam F, Dekker J, Tiana G, Heard E. Predictive polymer modeling reveals coupled fluctuations in chromosome conformation and transcription. Cell. 2014;157(4):950-63.

48. Frey BJ, Dueck D. Clustering by passing messages between data points. Science. 2007;315(5814):972-6.

49. Bodenhofer U, Kothmeier A, Hochreiter S. Apcluster: an R package for affinity propagation clustering. Bioinformatics. 2011;27(17):2463-4.

50. Bolstad BM, Irizarry RA, Åstrand M, Speed TP. A comparison of normalization methods for high density oligonucleotide array data based on variance and bias. Bioinformatics. 2003;19(2):185-93.

51. Bianco S, Lupiáñez DG, Chiariello AM, Annunziatella C, Kraft K, Schöpflin R, Wittler L, Andrey G, Vingron M, Pombo A, et al. Polymer physics predicts the effects of structural variants on chromatin architecture. Nat Genet. 2018;50(5):662.

52. Bintu B, Mateo LJ, Su J-H, Sinnott-Armstrong NA, Parker M, Kinrot S, Yamaya K, Boettiger AN, Zhuang X. Super-resolution chromatin tracing reveals domains and cooperative interactions in single cells. Science. 2018;362(6413):1783.

53. Consortium EP, et al. An integrated encyclopedia of dna elements in the human genome. Nature. 2012;489(7414):57.

54. Davis CA, Hitz BC, Sloan CA, Chan ET, Davidson JM, Gabdank I, Hilton JA, Jain K, Baymuradov UK, Narayanan AK, et al. The Encyclopedia of DNA elements (ENCODE): data portal update. Nucleic Acids Res. 2017;46(D1):794-801.

55. Breiman L. Random forests. Mach Learn. 2001;45(1):5-32.

56. Liaw A, Wiener $M$, et al. Classification and regression by randomforest. $R$ News. 2002;2(3):18-22.

57. Tjong H, Li W, Kalhor R, Dai C, Hao S, Gong K, Zhou Y, Li H, Zhou XJ, Le Gros MA, et al. Population-based 3D genome structure analysis reveals driving forces in spatial genome organization. Proc Natl Acad Sci. 2016;113(12):1663-72.

58. Hua N, Tjong H, Shin H, Gong K, Zhou XJ, Alber F. Producing genome structure populations with the dynamic and automated pgs software. Nat Protoc. 2018;13(5):915.

59. Nozawa R-S, Gilbert N. RNA: nuclear glue for folding the genome. Trends Cell Biol. 2019;29(3):201-211. https://doi.org/10.1016/j.tcb.2018.12.003.

60. Michieletto D, Gilbert N. Role of nuclear RNA in regulating chromatin structure and transcription. Curr Opin Cell Biol. 2019;58:120-5.

61. Kleiner A, Talwalkar A, Sarkar P, Jordan MI. A scalable bootstrap for massive data. J R Stat Soc Ser B Stat Methodol. 2014;76(4):795-816.

62. Sachs K, Perez O, Pe'er D, Lauffenburger DA, Nolan GP. Causal protein-signaling networks derived from multiparameter single-cell data. Science. 2005;308(5721):523-9.

63. Koller D, Friedman N. Probabilistic graphical models: principles and techniques. Cambridge: MIT press; 2009.

64. Boeva V, Louis-Brennetot C, Peltier A, Durand S, Pierre-Eugene C, Raynal V, Etchevers HC, Thomas S, Lermine A, Daudigeos-Dubus E, et al. Heterogeneity of neuroblastoma cell identity defined by transcriptional circuitries. Nat Genet. 2017;49(9):1408.

65. Pott S, Lieb JD. What are super-enhancers Nat Genet. 2015;47(1):8. 
66. Knight PA, Ruiz D. A fast algorithm for matrix balancing. IMA J Numer Anal. 2013;33(3):1029-47.

67. Durand NC, Shamim MS, Machol I, Rao SS, Huntley MH, Lander ES, Aiden EL. Juicer provides a one-click system for analyzing loop-resolution Hi-C experiments. Cell Syst. 2016;3(1):95-8.

68. Luce RD, Perry AD. A method of matrix analysis of group structure. Psychometrika. 1949;14(2):95-116.

69. Csardi G, Nepusz T, et al. The igraph software package for complex network research. InterJournal Complex Syst. 2006;1695(5):1-9.

70. Benjamini Y, Hochberg Y. Controlling the false discovery rate: a practical and powerful approach to multiple testing. J R Stat Soc Ser B Methodol. 1995;57(1):289-300.

71. Kuhn M, et al. Building predictive models in $\mathrm{R}$ using the caret package. J Stat Softw. 2008;28(5):1-26

72. Perez-Rathke A. Chromatin folder git repository. 2019. https://bitbucket. org/aperezrathke/chr-folder/. Accessed 12 Oct 2019.

73. Perez-Rathke A. CMX Gibbs sampler git repository. 2019. https:// bitbucket.org/aperezrathke/cmx/. Accessed 12 Oct 2019.

74. Perez-Rathke A. Many-body interaction loci scripts git repository. 2019. https://bitbucket.org/aperezrathke/chr-loc-mbi-mam. Accessed 12 Oct 2019.

75. Perez-Rathke A, Sun Q, Wang B, Boeva V, Shao Z, Liang J. CHROMATIX: computing the functional landscape of many-body chromatin interactions in transcriptionally active loci from deconvolved single-cells. http://doi.org/10.5281/zenodo.3553117.

76. Haeussler M, Zweig AS, Tyner C, Speir ML, Rosenbloom KR, Raney BJ, Lee CM, Lee BT, Hinrichs AS, Gonzalez JN, Gibson D, Diekhans M, Clawson H, Casper J, Barber GP, Haussler D, Kuhn RM, Kent W. The UCSC Genome Browser database: 2019 update. Nucleic Acids Res. 2018;47(D1):853-8. https://doi.org/10.1093/nar/gky1095.

77. Kent WJ, Sugnet CW, Furey TS, Roskin KM, Pringle TH, Zahler AM, Haussler D. The human genome browser at ucsc. Genome Res. 2002;12(6):996-1006.

78. Sing T, Sander O, Beerenwinkel N, Lengauer T. ROCR: visualizing classifier performance in R. Bioinformatics. 2005;21(20):3940-1.

\section{Publisher's Note}

Springer Nature remains neutral with regard to jurisdictional claims in published maps and institutional affiliations.

Ready to submit your research? Choose BMC and benefit from:

- fast, convenient online submission

- thorough peer review by experienced researchers in your field

- rapid publication on acceptance

- support for research data, including large and complex data types

- gold Open Access which fosters wider collaboration and increased citations

- maximum visibility for your research: over $100 \mathrm{M}$ website views per year

At BMC, research is always in progress.

Learn more biomedcentral.com/submissions 